\title{
ASYMPTOTIC DISTRIBUTIONS AND BERRY-ESSEEN INEQUALITIES FOR LOTKA-NAGAEV ESTIMATOR OF A POISSON RANDOMLY INDEXED BRANCHING PROCESS
}

\section{ZHENLONG GAO AND HUILI ZHANG}

Abstract. Consider a Galton-Watson process $\left\{Z_{n}\right\}$, the Lotka-Negaev estimator for offspring mean $m$ is $R_{n}=Z_{n+1} / Z_{n}$. Let $N_{t}$ be a Poisson process independent of $\left\{Z_{n}\right\}$, the continuous time process $\left\{Z_{N_{t}}\right\}$ is a Poisson randomly indexed branching process. We show the asymptotic distributions for $\left\{\mathbb{R}_{t}:=R_{N_{t}}\right\}$.

Mathematics subject classification (2010): $60 \mathrm{~J} 80$.

Keywords and phrases: Asymptotic distribution, Berry-Esseen's inequality, branching process, Poisson process.

\section{REFERENCES}

[1] K.B. AthreyA, Large deviation rates for branching processes. I: single type case, Ann. Appl. Probab., 4, 3 (1994), 779-790.

[2] W.J. CHU, Self-normalized large deviation for supercritical branching processes, J. Appl. Prob., 52 , 2 (2018), 450-458.

[3] J.P. DION AND T.W. EPPS, Stock prices as branching processes in random environments: estimation, Comm. Statist. Simulation Comput., 28, 4 (1999), 957-975.

[4] R. DurRett, Probabilty: theory and examples, 4rd edn., Cambridge University Press, New York, 2010.

[5] T.W. EPPS, Stock prices as branching processes, Stochastic Models, 12, 4 (1996), 529-558.

[6] K. Fleischmann And V. WACHTEL, Large deviations for sums indexed by the generations of a Galton-Watson process, Probab. Theory Relat. Fields, 141, 2 (2008), 455-470.

[7] Z.L. GAO, Berry-Esseen type inequality for a Poisson randomly indexed branching process via Stein's method, Journal of Mathematical Inequalities, 12, 2 (2018), 573-582.

[8] Z.L. GAO AND L.N. QIU, Large deviations for Lotka-Nagaev estimator of a randomly indexed branching process, Filomat, 32, 17 (2018), 5803-5808.

[9] I. GRAMA, Q.S. LiU AND E. MiQueU, Harmonic moments and large deviations for a supercritical branching process in a random environment, Electron. J. Probab, 22, (2017), 1-23.

[10] H. HE, On large deviation rates for sums associated with Galton-Watson processes, Adv. Appl. Probab., 48, 2 (2016), 672-690.

[11] J.N. LIU AND M. ZHANG, Large deviation for supercritical branching processes with immigration, Acta Mathematica Sinica, 32, 8 (2016), 893-900.

[12] S.V.NAGAEV, On estimating the expected number of direct descendants of a particle in a branching process, Theor. Probab. Appl., 12, 2 (1967), 314-320.

[13] P.E. NEY AND A.N. VIDYASHANKAR, Harmonic moments and large deviation rates for supercritical branching processes, Ann. Appl. Probab., 13, 2 (2003), 475-489.

[14] Q. SUn AND M. ZHANG, Harmonic moments and large deviations for supercritical branching processes with immigration, Front. Math. China, 12, 5 (2017), 1201-1220.

[15] S.J. WU, Large deviation results for a randomly indexed branching process with applications to $f$ nance and physics, Doctoral Thesis, Graduate Faculty of North Carolina State University, 2012. 